\title{
Preliminary Findings on the Use of Targeted Therapy in Combination with Sodium Phenylbutyrate in Advanced Malignant Mesothelioma: A Strategy for Improved Survival
}

\author{
Stanislaw R. Burzynski, Tomasz J. Janicki, Gregory S. Burzynski, Sheldon Brookman \\ Burzynski Clinic, Houston, TX, USA \\ Email: srb@burzynskiclinic.com
}

Received 23 August 2014; revised 20 September 2014; accepted 15 October 2014

Copyright (C) 2014 by authors and Scientific Research Publishing Inc.

This work is licensed under the Creative Commons Attribution International License (CC BY). http://creativecommons.org/licenses/by/4.0/

(c) (i) Open Access

\begin{abstract}
Advanced malignant mesothelioma (MM) is among the most aggressive and difficult-to-treat diseases. Industrialization and exposure to asbestos is the main causative factor for the dramatic increase in the incidence of MM, which carries a poor prognosis and a median survival of less than 12 months. Combination chemotherapy offers only palliative results; however, targeted therapy carries more promise for future successful treatment. This paper presents preliminary findings of improved overall survival $(O S)$ using a combination of sodium phenylbutyrate (PB) with various chemotherapeutic and targeted agents in advanced MM. The data suggest using a strategy of simultaneous interruption of signal transduction involving RAS-MEK-ERK, PI3K-AKT, mTOR, Merlin, and angiogenesis pathways and interference in cell cycle and epigenetic processes. Complete response was determined in $15.4 \%$ and stable disease in $46.2 \%$ in the group of 13 evaluable patients. Median OS for MM was higher compared to other treatments (17 months compared to between 6 and 12.1 months). The longest surviving patient continues to be in complete response and in excellent condition for over $\mathbf{1 2 . 5}$ years from the treatment start. These findings are only preliminary and validation of the results using a well-designed phase I/II trial in advanced MM is proposed.
\end{abstract}

\section{Keywords}

Mesothelioma, Mesothelioma Survival, Personalized Targeted Therapy, Antineoplastons, Sodium Phenylbutyrate, Clinical Studies 


\section{Introduction}

Advanced malignant mesothelioma (MM) is one of the most aggressive and difficult-to-treat neoplasms. It arises from the surface serosal cells of the pleura and peritoneum and in rare instances, the pericardium or gonads [1]. Exposure to asbestos fibers is the most common cause of MM. A very rare disease, industrialization and exposure to asbestos caused a dramatic increase in the incidence of MM once [2]. Further increase is expected, especially in developing nations [2]. The prognosis of MM is poor with the median survival from 9 to 12 months from diagnosis, despite advances in chemotherapy and use of targeted therapy [3]. Genetic analysis reveals involvement of a number of different signaling pathways and several important genetic alterations [1], which includes RAS-MEK-ERK, PI3K-AKT, m-TOR, Merlin, Hippo and angiogenesis pathways, interference in cell cycle and epigenetic changes. These mechanisms were described in detail in recent review articles [1] [4]-[7].

A combination of pemetrexed with cisplatin for unresectable pleural MM became the standard-of-care firstline chemotherapy [3]. Some targeted agents given as monotherapy or in combination, occasionally stabilize the disease, but none are recommended as standard first-line or second-line therapy [8].

For several years, our team has conducted research with antineoplastons (ANP), a group of anticancer agents consisting of peptides, amino acid derivatives, and carboxylic acids originally isolated from blood and urine of healthy subjects [9]-[11]. The anticancer activity of these compounds was confirmed in a number of preclinical and clinical studies [12]. After identification of the structure, numerous phase II studies were conducted with synthetic ANP A10 and ANP AS2-1 injections involving primary brain tumors and advanced colorectal cancer [13]-[18]. Further research revealed that some ingredients of ANP A10 and AS2-1, phenylacetylglutaminate (PG) and phenylacetate $(\mathrm{PN})$, are the same as metabolites of sodium phenylbutyrate $(\mathrm{PB})$ a histone deacetylase (HDAC) inhibitor with multiple targets of activity, which is approved for urea cycle disorders and indicated for glioma and acute promyelocytic leukemia [13] [19] [20]. The study of PG and PN on the glioblastoma multiforme (GBM) genome has shown that they affect approximately 100 genes [21]. These data and molecular profiling led to the treatment of a number of patients at Burzynski Clinic (BC) with advanced malignancies, including GBM and pancreatic cancer using combinations of PB and targeted agents [22] [23].

This article provides a brief description of treatment results in patients with advanced malignant mesothelioma and suggests a rationale for conducting clinical trials with PB in combination with targeted agents.

\section{Patients and Methods}

Thirteen subjects were diagnosed with advanced malignant mesothelioma following pathology and radiologic evaluations performed by independent institutions. Thereafter, treatment was provided in the private practice of the BC in Houston, TX. The patients included consecutively admitted evaluable patients between November 9, 2000 and August 24, 2012.

Blood and urine tests were done by the BC laboratory and by outside clinical laboratories. Tests were undertaken that included standard blood and urine analysis and determination of genomic markers (when these were available). Tissue molecular profiling was performed by Caris Life Sciences in Phoenix, AZ. All patients were required to read, understand, and sign an informed consent document prior to treatment. Treatment plans were formulated based on molecular profiling when obtained and included PB given alone or in combination with targeted and chemotherapeutic agents. Therapy was undertaken on an outpatient basis. After an initial two to four weeks at BC, treatment continued under the care of a local oncologist. Prior to the treatment start, a computerized tomography (CT) scan with and without contrast and in some patients a positron emission tomography (PET) scan was performed. The product of two of the largest perpendicular diameters (LPD) of the largest measurable lesions were calculated and totaled providing a baseline evaluation for each study subject. This baseline provided the reference for determining response outcome to treatment. Additional pretreatment measurements included vital signs, clinical disease status, demographics, medical history, current medications, physical examination, electrocardiogram (EKG) and Karnofsky Performance Status (KPS). The evaluation of toxicity was performed according to the common terminology criteria for adverse events version 3 (CTCAEv.3). Possible responses to treatment were complete response (CR), partial response (PR), stable disease (SD), or progressive disease (PD). CR required the disappearance of all lesions confirmed at the end of four weeks or a negative PET scan. PR required $50 \%$ or higher decrease of the LPD of measurable lesions confirmed at four weeks, PD was determined when new lesions appeared or when there was an increase over 25\% in the existing lesions and SD represented the status of tumors classified as between PR and PD. 


\section{Results}

\subsection{Patient Demographics}

The characteristics of the 13 patients studied are described in Table 1. All had involvement of the pleura, and seven patients had additional involvement of the peritoneum. Of all the patients, one had no prior treatment, whereas four patients had undergone surgery as their only treatment. The majority of patients had failed prior chemotherapy and one patient failed surgery and radiation therapy (RT). The data confirming diagnosis, recurrence, and response to treatment received are described in Table 2(a) and Table 2(b).

\subsection{Treatment}

Details of medication dosing and treatment duration of patients who obtained CR and SD are described in Table 3(a) and Table 3(b).

Five patients received treatment with the single agent PB. Five additional patients were treated with $\mathrm{PB}$ and erlotinib-Genentech/Astellas Pharma US/OSI Pharmaceuticals. In four of these, treatment was combined with multikinase inhibitors (sorafenib-Bayer and Onyx Pharmaceuticals, pazopanib—GlaxoSmithKline, or sunitinib— Pfizer). One of five patients that were treated with a combination that included erlotinib was also treated with bevacizumab (BVZ) - Genentech/Roche and chemotherapy consisting of pemetrexate-Eli Lilly and Company, and cisplatin-Bristol-Myers Squibb Company. Lastly, one patient who received erlotinib for a short period of time along with lapatinib-GlaxoSmithKline was also treated with the chemotherapy agent nab-paclitaxelAbraxis BioScience/Celgene Corporation.

\subsection{Responses and Survival}

CR, SD and PD was achieved in $15.4 \%(\mathrm{~N}=2), 46.2 \%(\mathrm{~N}=6)$ and $38.4 \%(\mathrm{~N}=5)$, respectively. Of the five patients who received treatment with PB as monotherapy, one obtained CR, two exhibited SD and two developed PD. Two patients are currently alive. One of these patients who were receiving PB monotherapy (Patient 1) experienced a remarkably prolonged OS in excess of 12.5 years after having failed three lines of chemotherapy.

Table 1. Demographics of patients with advanced malignant mesothelioma.

\begin{tabular}{|c|c|c|c|}
\hline Characteristic & & $\mathrm{N}=13$ & $\%$ \\
\hline \multirow[t]{3}{*}{ Age (year) } & & $\mathrm{N}$ & $\%$ \\
\hline & Median & 69 & \\
\hline & Range & $41-79$ & \\
\hline \multicolumn{4}{|l|}{ Sex } \\
\hline & Male & 9 & 69 \\
\hline & Female & 4 & 31 \\
\hline \multicolumn{4}{|c|}{ KPS (Karnofsky performance status score) } \\
\hline & 90 & 4 & 31 \\
\hline & 80 & 2 & 15 \\
\hline & 70 & 3 & 23 \\
\hline & 60 & 1 & 8 \\
\hline & 50 & 3 & 23 \\
\hline \multicolumn{4}{|l|}{ Location } \\
\hline & Pleura & 6 & 46 \\
\hline & Pleura and peritoneum & 7 & 54 \\
\hline \multicolumn{4}{|l|}{ Prior treatment } \\
\hline & No prior treatment & 1 & 8 \\
\hline & Surgery only & 4 & 31 \\
\hline & Surgery and RT & 1 & 8 \\
\hline & Chemotherapy only & 2 & 15 \\
\hline & Surgery and chemotherapy & 5 & 38 \\
\hline
\end{tabular}


Table 2. (a) Confirmation of diagnosis, recurrence, treatment and response; (b) Confirmation of diagnosis, progression and response-mesothelioma, newly-diagnosed.

(a)

\begin{tabular}{|c|c|c|c|c|c|c|c|c|c|c|}
\hline \multicolumn{4}{|c|}{ Confirmation of Diagnosis } & \multirow{3}{*}{ Treatment } & \multirow{2}{*}{\multicolumn{2}{|c|}{$\begin{array}{l}\text { Confirmation of } \\
\text { Recurrence }\end{array}$}} & \multirow{2}{*}{\multicolumn{3}{|c|}{$\begin{array}{l}\text { Confirmation of } \\
\text { Response to PBT }\end{array}$}} & \multirow{3}{*}{ 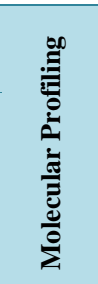 } \\
\hline \multicolumn{2}{|c|}{ Pathology } & \multicolumn{2}{|c|}{ Radiology } & & & & & & & \\
\hline 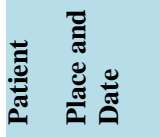 & 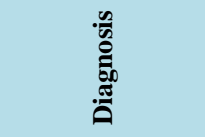 & 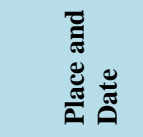 & 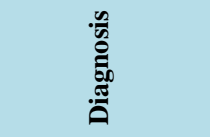 & & 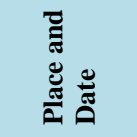 & 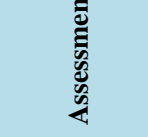 & 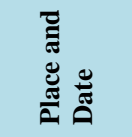 & 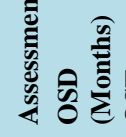 & 它 & \\
\hline \multicolumn{11}{|c|}{ RECURRENT (PERSISTENT) AFTER CHEMOTHERAPY AND RADIATION THERAPY } \\
\hline \multirow[t]{4}{*}{$\begin{array}{c}1 \text { University } \\
\text { hospital } \\
\text { August } \\
\text { 16, } 2001\end{array}$} & $\begin{array}{c}\text { Malignant } \\
\text { mesothelioma } \\
\text { right pleural } \\
\text { effusion positive } \\
\text { for malignancy }\end{array}$ & $\begin{array}{c}\text { University } \\
\text { hospital CT } \\
\text { August 8, } \\
2001\end{array}$ & $\begin{array}{l}\text { Omental } \\
\text { nodularities, } \\
\text { ascites, } \\
\text { pleural } \\
\text { effusion }\end{array}$ & $\begin{array}{c}\text { Exploratory } \\
\text { laparoscopy, } \\
\text { partial } \\
\text { omentectomy, } \\
\text { total abdominal } \\
\text { hysterectomy } \\
\text { and bilateral } \\
\text { salpingo- } \\
\text { oophorectomy } \\
\text { August 10, } 2001\end{array}$ & & & & $\begin{array}{c}157.3 \\
+\end{array}$ & $\begin{array}{c}150.2 \\
+\end{array}$ & \\
\hline & & & & $\begin{array}{c}\text { Paclitaxel and } \\
\text { carboplatin x3 } \\
\text { August 21, } \\
\text { 2001- } \\
\text { October 3, } \\
2001 \\
\text { Topotecan } \\
\text { to February } \\
\text { 29, } 2002\end{array}$ & $\begin{array}{c}\text { Regional } \\
\text { radiology } \\
\text { CT } \\
\text { November } \\
\text { 7, } 2001\end{array}$ & Recurrence & & & & \\
\hline & & & & $\begin{array}{l}\text { Thalidomide } \\
\text { to March } \\
12,2002\end{array}$ & $\begin{array}{c}\text { Regional } \\
\text { radiology } \\
\text { March 12, } \\
2002\end{array}$ & $\begin{array}{l}\text { Persistent } \\
\text { disease }\end{array}$ & & & & \\
\hline & & & $\begin{array}{c}\text { Stage at } \\
\text { admission } \\
\text { to BC, Stage } \\
\text { IV pleural and } \\
\text { peritoneal } \\
\text { malignant } \\
\text { mesothelioma }\end{array}$ & $\begin{array}{l}\text { BC. March } \\
\text { 19, 2002. PB }\end{array}$ & & & $\begin{array}{l}\text { Regional } \\
\text { radiology } \\
\text { PET } \\
\text { December } \\
\text { 6, } 2002 \\
\text { and March } \\
\text { 14, } 2003\end{array}$ & CR & & \\
\hline \multirow[t]{2}{*}{$\begin{array}{c}2 \text { University } \\
\text { hospital } \\
\text { May 4, } \\
2005\end{array}$} & $\begin{array}{c}\text { Malignant } \\
\text { mesothelioma }\end{array}$ & $\begin{array}{l}\text { Regional } \\
\text { radiology } \\
\text { CT April } \\
18,2005\end{array}$ & $\begin{array}{l}\text { Mass within } \\
\text { the left lower } \\
\text { lobe, mediastinal } \\
\text { lymph nodes, } \\
\text { left pleural } \\
\text { effusion }\end{array}$ & $\begin{array}{l}\text { Decortication } \\
\text { and pleurodesis } \\
\text { May 5, } 2005 \text {. } \\
\text { RT × } 3 \text { days }\end{array}$ & $\begin{array}{c}\text { Regional } \\
\text { radiology } \\
\text { CT } \\
\text { September } \\
\text { 21, } 2005\end{array}$ & Recurrence & & 13.8 & 9.0 & \\
\hline & & & $\begin{array}{c}\text { Stage at } \\
\text { admission to } \\
\text { BC: Stage IV, } \\
\text { Pleural malignant } \\
\text { mesothelioma }\end{array}$ & $\begin{array}{l}\text { BC. September } \\
\text { 29, } 2005 \text { PB, } \\
\text { erlotinib, } \\
\text { imatinib, } \\
\text { sorafenib, } \\
\text { methotrexate. }\end{array}$ & & & $\begin{array}{c}\text { Regional } \\
\text { radiology } \\
\text { CT } \\
\text { November } \\
\text { 25, } 2005\end{array}$ & $\mathrm{PD}$ & & $\begin{array}{l}\text { VEGF } \\
\text { and } \\
\text { EGFR- } \\
\text { elevated } \\
\text { (blood) }\end{array}$ \\
\hline $\begin{array}{c}3 \text { University } \\
\text { hospital } \\
\text { July 6, } \\
2006\end{array}$ & $\begin{array}{c}\text { Malignant } \\
\text { mesothelioma }\end{array}$ & $\begin{array}{l}\text { Regional } \\
\text { medical } \\
\text { center CT } \\
\text { June 15, } \\
2006\end{array}$ & $\begin{array}{l}\text { Very large left } \\
\text { pleural effusion. } \\
\text { Large mass } \\
\text { involving the } \\
\text { left lateral } \\
\text { aspects of the } \\
\text { arch of aorta. }\end{array}$ & $\begin{array}{l}\text { Pemetrexed } \\
\text { July 18, } 2006\end{array}$ & $\begin{array}{c}\text { Regional } \\
\text { radiology } \\
\text { CT/PET } \\
\text { August 1, } \\
2006\end{array}$ & $\begin{array}{l}\text { Persistent } \\
\text { disease }\end{array}$ & & 40.0 & 27.6 & \\
\hline
\end{tabular}




\section{Continued}

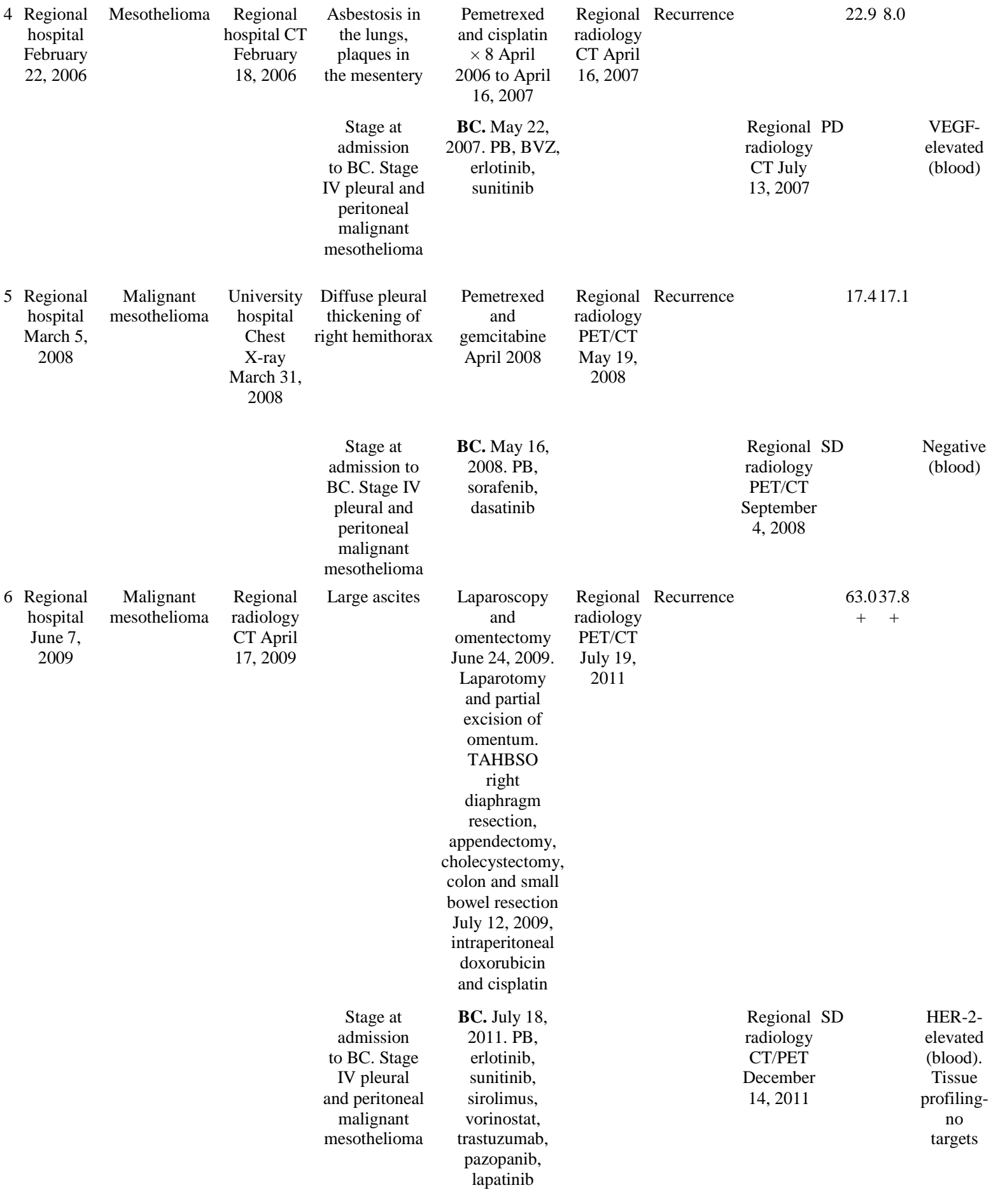




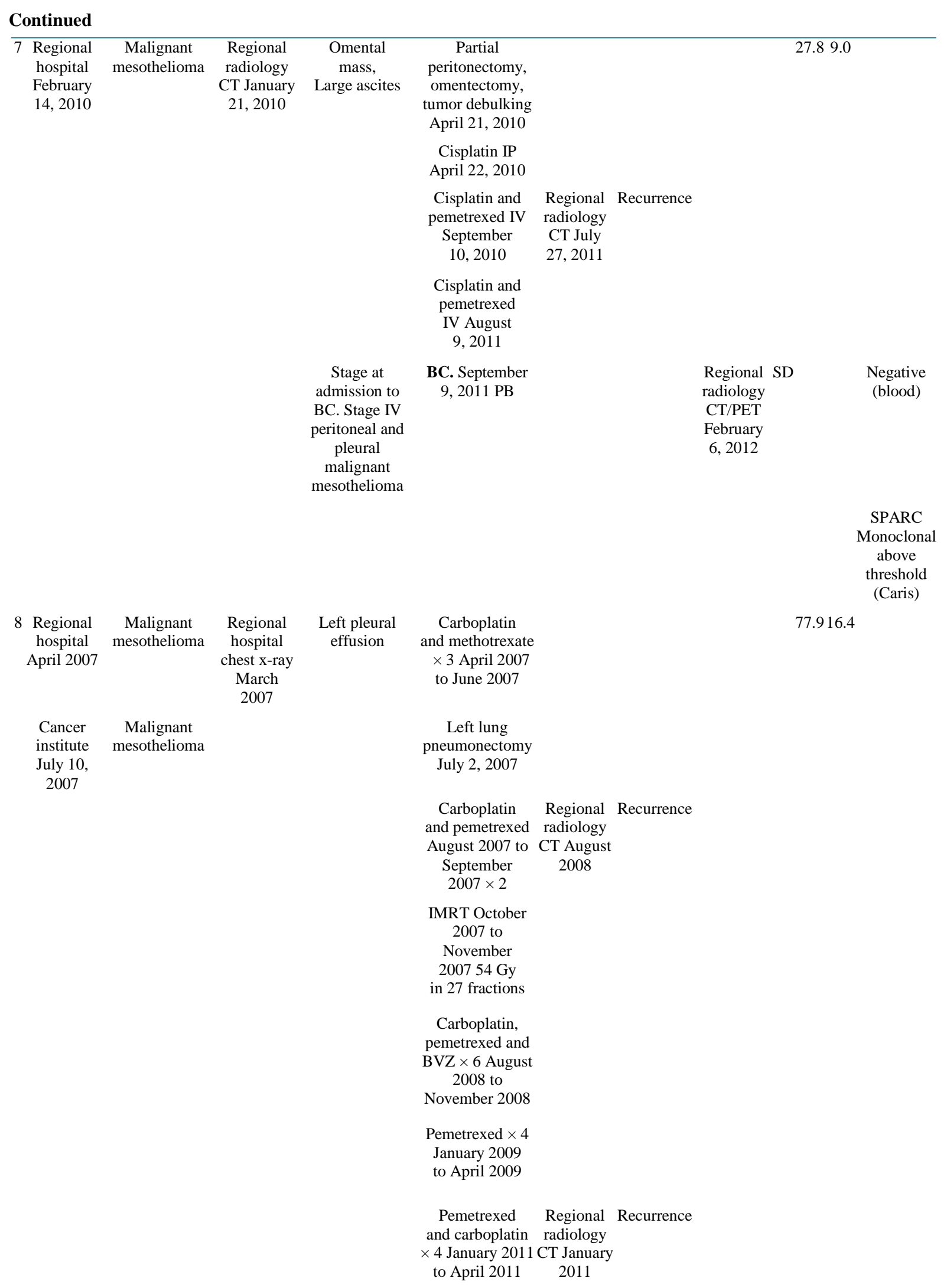




\section{Continued}

Stage at admission to BC. Stage IV pleural malignant mesothelioma

\begin{tabular}{|c|c|c|}
\hline $\begin{array}{c}\text { Chemo } \\
\text { embolization } \times 10 \\
\text { April } 2011 \text { to } \\
\text { December } 2011 \\
\text { with gemcitabine } \\
\text { cisplatin, and } \\
\text { mitomycin }\end{array}$ & & \\
\hline 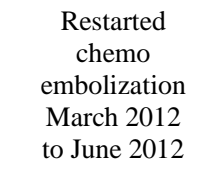 & $\begin{array}{c}\text { Regional } \\
\text { radiology } \\
\text { CT March } \\
2012\end{array}$ & Recurrence \\
\hline $\begin{array}{c}\text { Pemetrexed } \\
\text { and BVZ × } 1 \\
\text { August } 1,2012\end{array}$ & $\begin{array}{l}\text { Regional } \\
\text { radiology } \\
\text { MRI June } \\
28,2012\end{array}$ & Recurrence \\
\hline
\end{tabular}

BC. August 24, 2012 PB, pazopanib, everolimus

$\begin{array}{cc}\begin{array}{c}\text { Regional PD } \\ \text { radiology } \\ \text { CT }\end{array} & \begin{array}{c}\text { VEGF- } \\ \text { elevated } \\ \text { January }\end{array} \\ \text { (blood), } \\ \text { BRC, } 2013 & \text { ERCC1, } \\ & \text { TS, RR1- } \\ & \text { low, } \\ & \text { MGMT- } \\ & \text { negative, } \\ & \text { SPARC } \\ & \text { mono } \\ & \text { clonal- } \\ & \text { positive } \\ & \text { (tissue, }\end{array}$

Caris)

Abbreviations: BC—Burzynski clinic; BRCA1 — breast cancer type 1 susceptibility gene; BVZ—bevacizumab; CT — computed tomography; EGFR— epidermal growth factor receptor; ERCC1 - excision repair cross-complementation group 1 enzyme; HER2-human epidermal growth factor receptor 2; IMRT — intensity-modulated radiation therapy; MGMT-O-6-methylguanine-DNA methyltransferase; MRI-magnetic resonance imaging; OSD — overall survival from diagnosis; OST—overall diagnosis from treatment start; PB — sodium phenylbutyrate; PBT_-PB and other drugs; PD — progressive disease; PET—positron emission tomography; RR1—ribonucleotide reductase; RT—radiation therapy; SD—stable disease; SPARC— secreted protein acidic and rich in cysteine; TAHBSO — total abdominal hysterectomy bilateral saphingo-oophorectomy; TS —-thymidylate synthase enzyme; VEGF — vascular endothelial growth factor; +- - patient is still alive.

(b)

\begin{tabular}{|c|c|c|c|c|c|c|c|c|c|c|c|c|}
\hline \multicolumn{5}{|c|}{ Confirmation of Diagnosis } & \multirow{3}{*}{ Treatment } & \multirow{2}{*}{\multicolumn{2}{|c|}{$\begin{array}{l}\text { Confirmation } \\
\text { of Progression }\end{array}$}} & \multirow{2}{*}{\multicolumn{3}{|c|}{$\begin{array}{c}\text { Confirmation of } \\
\text { Response } \\
\text { to PBT }\end{array}$}} & \multirow{3}{*}{\multicolumn{2}{|c|}{$\begin{array}{c}\text { Molecular } \\
\text { Profiling }\end{array}$}} \\
\hline \multicolumn{3}{|c|}{ Pathology } & \multicolumn{2}{|c|}{ Radiology } & & & & & & & & \\
\hline • & 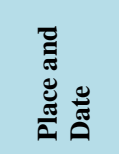 & 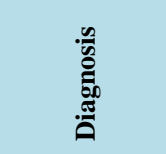 & \multicolumn{2}{|r|}{ 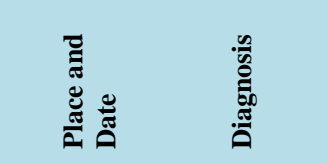 } & & 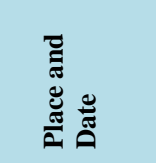 & 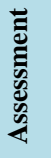 & \multirow{2}{*}{\multicolumn{2}{|c|}{ 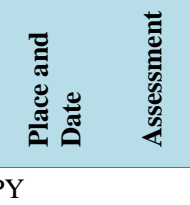 }} & ด & & \\
\hline \multicolumn{11}{|c|}{ NO PRIOR CHEMOTHERAPY OR RADIATION THERAPY } & & \\
\hline \multirow[t]{3}{*}{9} & $\begin{array}{c}\text { University } \\
\text { hospital } \\
\text { January } \\
20,2000\end{array}$ & $\begin{array}{c}\text { Malignant } \\
\text { mesothelioma }\end{array}$ & $\begin{array}{l}\text { Regional } \\
\text { radiology } \\
\text { CT February } \\
24,2000\end{array}$ & $\begin{array}{l}\text { Right pleural } \\
\text { thickening }\end{array}$ & $\begin{array}{c}\text { Pleurodesis } \\
\text { January 20, } \\
2000\end{array}$ & & & & & 14.6 & 5.1 & \\
\hline & & & & & & $\begin{array}{c}\text { University } \\
\text { hospital CT } \\
\text { November 3, } \\
2000\end{array}$ & $\mathrm{PD}$ & & & & & \\
\hline & & & & $\begin{array}{c}\text { Stage at } \\
\text { admission } \\
\text { to BC. Stage } \\
\text { IV malignant } \\
\text { pleural } \\
\text { mesothelioma }\end{array}$ & $\begin{array}{l}\text { BC. November } \\
\text { 9, 2000. PB }\end{array}$ & & & $\begin{array}{c}\text { University } \\
\text { hospital } \\
\text { January } 8 \\
2001\end{array}$ & y & & & None \\
\hline
\end{tabular}




\section{Continued}

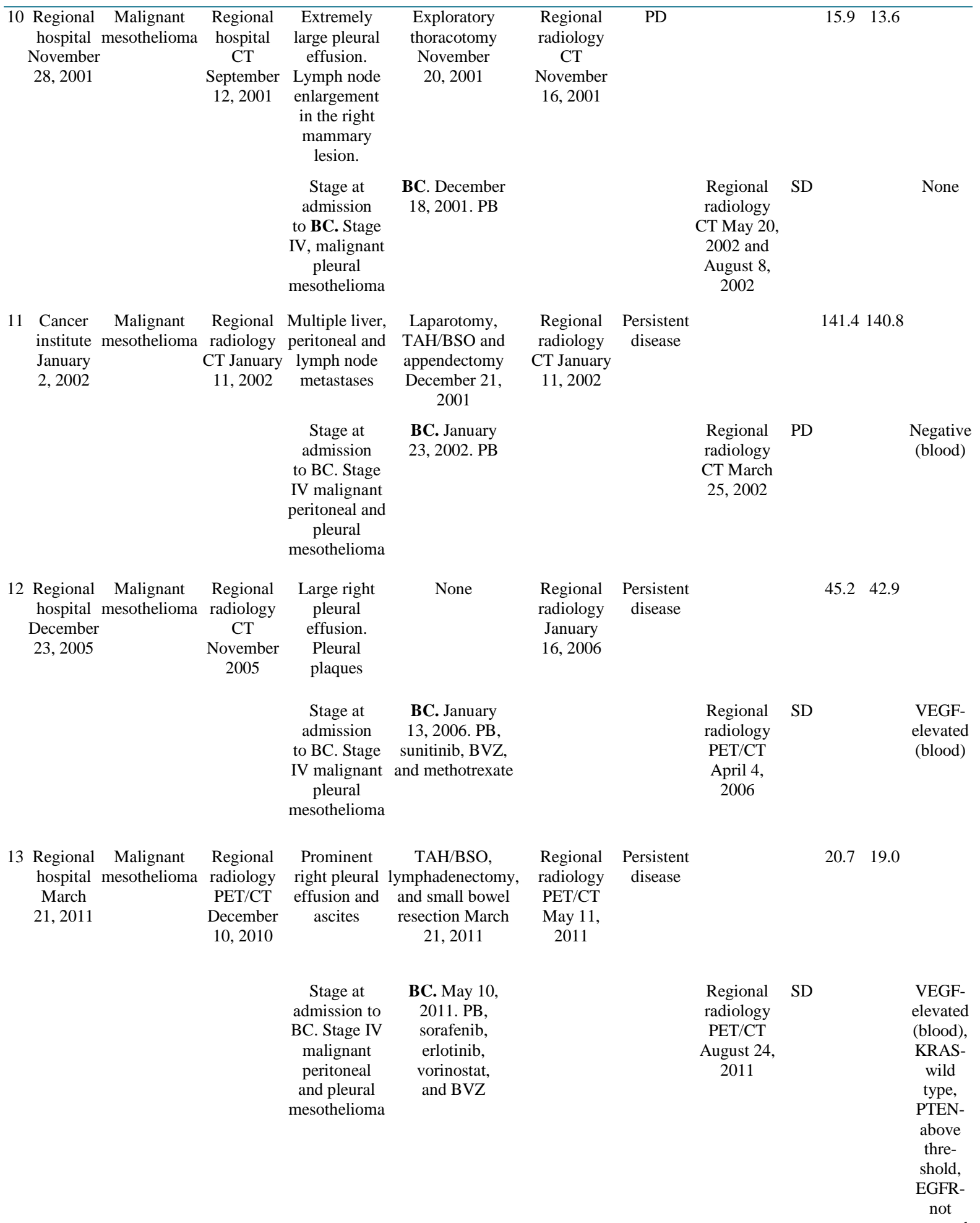

mutated

Abbreviations: BC—Burzynski clinic; BVZ—bevacizumab; CT—computed tomography; EGFR—epidermal growth factor receptor; KRAS— Kirsten rat sarcoma viral oncogene homolog; MRI—magnetic resonance imaging; OSD—overall survival from diagnosis; OST—overall diagnosis from treatment start; PB — sodium phenylbutyrate; PBT—PB and other drugs; PD—progressive disease; PET—positron emission tomography; PTEN— phosphatase and tensin homolog; SD—-stable disease; TAH/BSO — total abdominal hysterectomy bilateral saphingo—oophorectomy; VEGF— vascular endothelial growth factor 
Table 3. (a) Medication dose and duration of treatment until first response; (b) Medication dose and duration of treatment until first response.

(a)

\begin{tabular}{|c|c|c|c|c|c|c|c|c|c|}
\hline \multirow{2}{*}{ Patient } & \multicolumn{9}{|c|}{ Targeted Drugs Daily Dose/Duration } \\
\hline & PB & Erlotinib & Pazopanib & Sorafenib & Sunitinib Sirolimus & Dasatinib & Vorinostat & Lapatinib & Bevacizumab Trastuzumab \\
\hline 1 & $24 \mathrm{~g} / 8.5 \mathrm{~m}$ & & & & & & & & \\
\hline 3 & $15 \mathrm{~g} / 4.5 \mathrm{~m}$ & $100 \mathrm{mg} / 3 \mathrm{~m}$ & & & & & & & $\begin{array}{c}5 \mathrm{mg} / \mathrm{kg} \times 6 \\
10 \mathrm{mg} / \mathrm{kg} \times 2\end{array}$ \\
\hline 5 & $12.5 \mathrm{~g} / 4 \mathrm{~m}$ & & & $400 \mathrm{mg} / 3.5 \mathrm{~m}$ & & $50 \mathrm{mg} / 3 \mathrm{~m}$ & & & \\
\hline 6 & $12 \mathrm{~g} / 4.5 \mathrm{~m}$ & $150 \mathrm{mg} / 0.5 \mathrm{n}$ & $200 \mathrm{mg} / 1 \mathrm{~m}$ & & $25 \mathrm{mg} / 4 \mathrm{~m} 1 \mathrm{mg} / 1 \mathrm{~m}$ & & $100 \mathrm{mg} / 1 \mathrm{~m}$ & $\begin{array}{l}750 \mathrm{mg} / \\
3.5 \mathrm{~m}\end{array}$ & $2 \mathrm{mg} / \mathrm{kg} \times 2$ \\
\hline 7 & $12 \mathrm{~g} / 5 \mathrm{~m}$ & & & & & & & & \\
\hline 10 & $30 \mathrm{~g} / 5 \mathrm{~m}$ & & & & & & & & \\
\hline 12 & $18 \mathrm{~g} / 3 \mathrm{~m}$ & & & & & & & & $10 \mathrm{mg} / \mathrm{kg} \times 8$ \\
\hline 13 & $12 \mathrm{~g} / 3.5 \mathrm{~m}$ & $150 \mathrm{mg} / 4 \mathrm{~m}$ & & $200 \mathrm{mg} / 7 \mathrm{~m}$ & & & $100 \mathrm{mg} / 7 \mathrm{~m}$ & & $2.5 \mathrm{mg} / \mathrm{kg} \times 6$ \\
\hline
\end{tabular}

(b)

\begin{tabular}{cccc}
\hline \multirow{2}{*}{ Patient } & & Cytotoxic Chemotherapy Daily Dose/Duration & \\
\cline { 2 - 4 } & Pemetrexed & Cisplatin & Nab-paclitaxel \\
\hline 3 & $500 \mathrm{mg} / \mathrm{m}^{2} \times 5$ & $60 \mathrm{mg} / \mathrm{m}^{2} \times 5$ & \\
6 & & & $100 \mathrm{mg} / \mathrm{m}^{2} \times 2$ \\
\hline
\end{tabular}

Currently, she continues to be classified as CR and remains disease-free. The second patient who achieved CR (Patient 3) had received treatment with PB plus erlotinib, BVZ, pemetrexate, trastuzumab-Genentech, and cisplatin (cf. Figure 1). The remaining cases of SD were treated with a combination of PB plus erlotinib, multikinase inhibitors, and BVZ. One of these patients also received chemotherapy with nab-paclitaxel. The median OS in this evaluation was 17 months and is compared to the other studies, as described in Table 4. Survival was measured from the first day of administration of therapy at Burzynski Clinic until death from any cause, and time to treatment failure was likewise measured from the first day of the treatment until the date of first observation of progressive disease or death from any cause whichever came first. The distributions of survival and treatment failure were estimated by Kaplan-Meier analysis. OS compares favorably to the other studies with chemotherapy for advanced malignant mesothelioma. The Kaplan-Meier Survival Curves are presented in Figure 2 and Figure 3 (for all patients and for eight patients with recurrent MM, respectively). The Kaplan-Meier analysis was prepared by using the MedCalc Statistical Software version 13.3 (MedCalc Software bvba, Ostend, Belgium; 2014).

\subsection{Safety and Adverse Events}

Grades 3 and 4 adverse drug events (ADEs) described in Table 5 were compared with results in other trials. The most common ADEs noted according to CTCAEv.3 were infections and diarrhea. Grade 2 ADEs are not included based upon a paucity of data in several of the comparator studies. Among the Grade 2 ADEs that were observed in this evaluation, most were nausea and vomiting, diarrhea, rash, fatigue and anorexia. These ADEs were readily reversible within a short time.

\section{Discussion}

For over ten years, standard first-line therapy for advanced MM includes combination chemotherapy with pemetrexate and cisplatin [3]. Additional chemotherapy regimens have been proposed including mitomycin-C plus vinblastine and cisplatin, and cisplatin plus gemcitabine, but the response rate and OS was lower compared to 


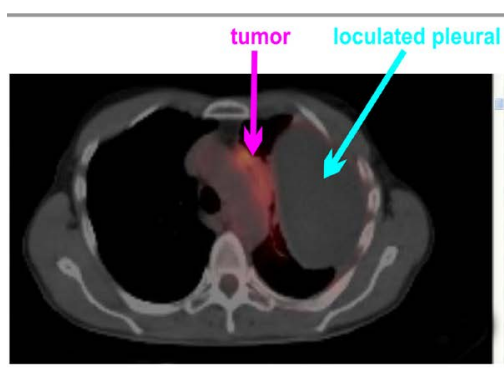

Aug. 1, 2006 Baseline PET/CT

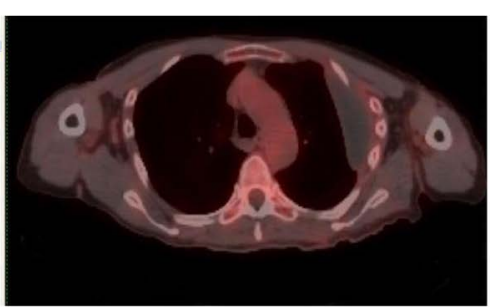

Dec. 5, 2006 Follow-up PET/CT

Figure 1. A complete response of pleural malignant mesothelioma; patient 3 PET/CT scan indicating a complete resolution of the tumor.

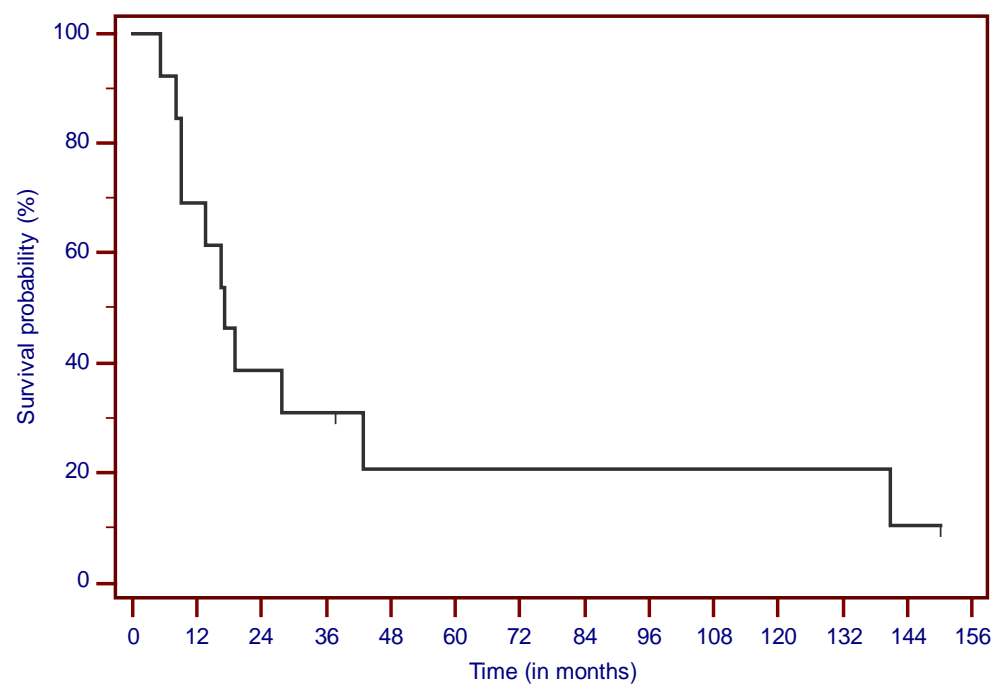

Figure 2. The Kaplan-Meier survival curve. Overall survival from the treatment start for all patients.

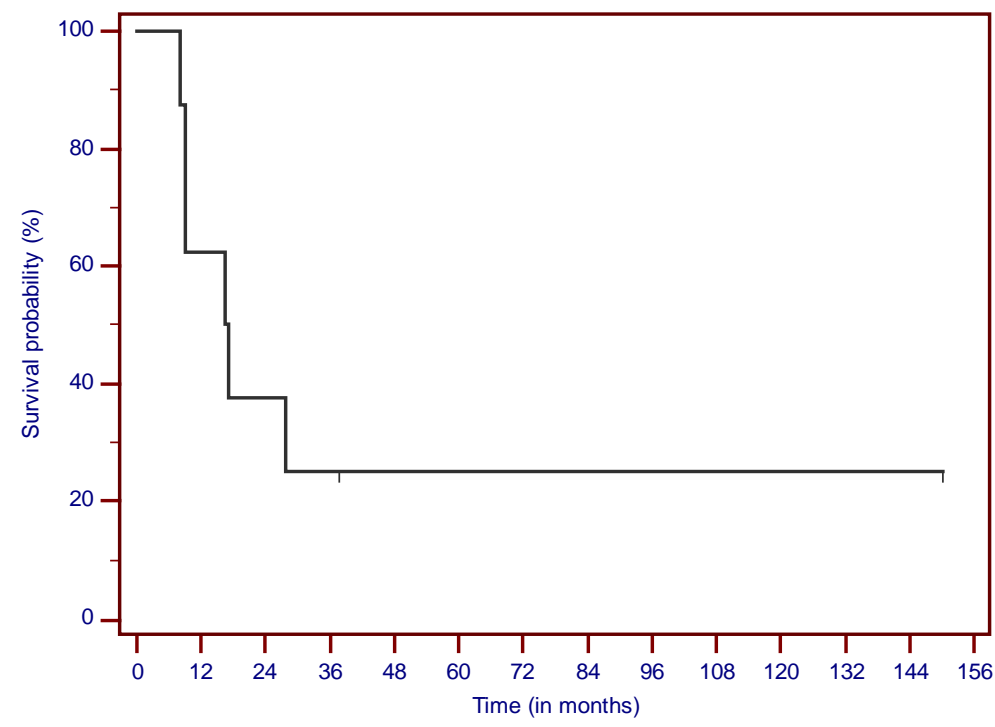

Figure 3. The Kaplan-Meier survival curve. Overall survival from the treatment start for patients with recurrent MM. 
Table 4. Selected clinical studies in advanced malignant mesothelioma.

\begin{tabular}{cccc}
\hline Reference & Treatment & Number of patients & Median OS (months) \\
\hline Middleton et al. $1998^{24}$ & Mitomycin-C, vinblastine + cisplatin & 39 & 6 \\
Nowak et al. $2008^{25}$ & Cisplatin + gemcitabine & 53 & 11.2 \\
Vogelzang et al. $2003^{3}$ & Pemetrexed + cisplatin & 226 & 12.1 \\
Vogelzang et al. $2003^{3}$ & Cisplatin & 222 & 9.3 \\
Burzynski et al. 2014 & PB + targeted combination & 13 & 17 \\
\hline
\end{tabular}

Abbreviations: OS—overall survival; PB—sodium phenylbutyrate.

Table 5. Incidence of adverse drug events (ADEs), grades 3 and 4 in second-line therapy of patients with advanced mesothelioma.

\begin{tabular}{|c|c|c|c|c|c|c|c|c|}
\hline \multirow{4}{*}{ ADE (incidence \%) } & \multicolumn{8}{|c|}{ REFERENCES } \\
\hline & \multirow{2}{*}{\multicolumn{2}{|c|}{$\begin{array}{c}\text { Middleton et al. } \\
1998^{24} \\
\text { Grades }\end{array}$}} & \multicolumn{2}{|c|}{ Nowak et al. $2008^{25}$} & \multirow{2}{*}{$\begin{array}{c}\text { Vogelzang et al. } \\
2003^{3} \text { Pemetrexed + } \\
\text { Cisplatin } 226 \\
\text { Grades }\end{array}$} & \multirow{2}{*}{$\begin{array}{c}\text { Vogelzang et al. } \\
2003^{3} \text { Cisplatin } \\
\text { Grades }\end{array}$} & \multicolumn{2}{|c|}{$\begin{array}{c}\text { Burzynski et al. } \\
2014\end{array}$} \\
\hline & & & & & & & & \\
\hline & 3 & 4 & 3 & 4 & $3 / 4$ & $3 / 4$ & 3 & 4 \\
\hline \multicolumn{9}{|l|}{ General } \\
\hline Fatigue & & & & & 10.2 & 8.6 & 7.7 & \\
\hline Fever & & & & & 1.3 & & & \\
\hline Infection & 5.4 & - & 4 & - & 1.3 & 0.5 & 15.4 & \\
\hline \multicolumn{9}{|l|}{ Hematologic } \\
\hline Hemoglobin & 2.7 & - & 7 & - & 4.8 & & & \\
\hline Leukopenia & 10.8 & 2.7 & 30 & 6 & 17.7 & 0.9 & 7.7 & \\
\hline Lymphopenia & & & & & & & & \\
\hline Neutropenia & & & 32 & 24 & 27.9 & 2.3 & 7.7 & \\
\hline Thrombocytopenia & 2.7 & - & 17 & 32 & 5.8 & & & 7.7 \\
\hline \multicolumn{9}{|l|}{ Gastrointestinal } \\
\hline Anorexia & & & & & 2.2 & 0.5 & 7.7 & \\
\hline Constipation & 2.7 & - & & & & & & \\
\hline Diarrhea & - & - & 2 & - & 4.4 & & 15.4 & \\
\hline Dyspepsia & & & & & & & & \\
\hline Dehydration & & & & & 4.0 & 0.5 & & \\
\hline Mucositis & - & - & & & & & 7.7 & \\
\hline Nausea/vomiting & 8.1 & - & $20 / 17$ & - & 14.6/13.3 & $6.3 / 3.6$ & $-/ 7.7$ & \\
\hline Stomatitis & & & - & - & 4 & & & \\
\hline \multicolumn{9}{|l|}{ Cardiovascular } \\
\hline Hypertension & & & & & & & 7.7 & \\
\hline Chest pain & & & & & & & 7.7 & \\
\hline \multicolumn{9}{|l|}{ Neurologic } \\
\hline Paresthesia & & & & & & & 7.7 & \\
\hline Other & & & 13 & - & & & & \\
\hline \multicolumn{9}{|l|}{ Dermatologic } \\
\hline Rash & & & & & 1.3 & & & \\
\hline \multicolumn{9}{|l|}{ Metabolic } \\
\hline Hypokalemia & & & & & & & 7.7 & \\
\hline
\end{tabular}

Toxicity criteria: WHO-Middleton; CTCAE v.2-Nowak; CTCAE V.3-Burzynski. 
the pemetrexate-cisplatin combination [24] [25]. Unfortunately, after failure of first-line chemotherapy, the standard therapy has yet to be recommended [26]. Numerous targeted therapy regimens have been studied and results summarized in recent reviews [8].

After some success in treating non-small-cell carcinoma of the lung with tyrosine kinase inhibitors (TKI) gefitinib and erlotinib, use of these agents in MM appeared reasonable. Most MM tumors (68\%) expressed epidermal growth factor receptor (EGFR), but contrary to lung cancer, the activating mutations of EGFR are rare in MM [27] [28]. This may explain the failure of erlotinib in phase II trials in MM used as a single agent or in combination with BVZ [29] [30]. Platelet derived growth factor (PDGF) plays an important part in MM pathogenesis, but the PDGF inhibitor imatinib failed to produce objective responses in phase II trials [31] [32]. PR was shown in 2\% of SRC and PDGF inhibitor dasatinib patients, but patients developed serious toxicity [33]. Inhibition of angiogenesis was another important aspect of the activity of MM. Monoclonal antibody against vascular endothelial growth factor type 2 (VEGF2) and BVZ in combination with cisplatin and pemetrexate, used as a first-line regimen, produced a $43 \%$ response rate [34]. Multitargeted TKIs, both sunitinib and sorafenib, produced responses and a median OS of 6.7 and 10.7 months, respectively and pazopanib is currently under investigation [35] [36]. Histone deacetylase inhibitors have also been tested in clinical studies in MM. Vorinostat is most advanced of these studies and has shown PRs in an early phase I trial. Based upon these results, a phase III trial has been initiated, but was not successful [37].

The findings reported in this paper are derived from the treatment of consecutively admitted evaluable MM patients in private practice at BC. The majority of these cases had already failed standard treatment and were given little, if any, hope by their prior treating physicians. The choice of targeted agents and molecular profiling was very limited when the initial patients began treatment and for this reason, they were treated only with the HDAC inhibitor, PB.

As indicated by our studies on the effect of PB metabolites on the neoplastic genome, the PB treatment may affect over 100 genes instrumental in the promotion of malignant growth [38]. Knowing that the effect of PB is not very strong, we were surprised when one out of five patients on PB monotherapy responded with a long period of complete response and survival in excess of 12.5 years. Also of note is that this patient had disease recurrence after three lines of chemotherapy prior to PB. Another patient who obtained a CR was previously treated with pemetrexed, which was continued under our care in combination with PB, erlotinib, BVZ, and cisplatin. Two additional patients obtained SD on PB monotherapy and two other patients obtained SD as the result of a combination of $\mathrm{PB}$, erlotinib plus the multikinase inhibitor, sorafenib or pazopanib. One additional patient had SD after treatment with the combination of PB, sorafenib, and dasatinib. Following is a discussion of the emerging strategy for the successful treatment of advanced malignant mesothelioma.

Carcinogenesis induced by asbestos fibers in MM is discussed in numerous articles [4]-[7]. Through a number of different mechanisms, asbestos fibers induce genetic damage and activate signaling networks in mesothelial and stromal cells and macrophages that support transformation and maintenance of the neoplastic process. RAF-MEK-ERK and PI3K-AKT pathway activation plays an important part in this process and can be triggered upstream by several RTK's including EGFR, PDGF, and metastatic oncogene receptor, (MET) [27] [28] [39]. One of the downstream targets of PI3K-AKT pathways is mammalian target of rapamycin (mTOR). Simultaneous activation of PI3K-AKT and mTOR signaling was associated with reduced survival in MM [40]. Overactive SRC kinases can also play an important part in progression of MM indicating the therapeutic application of dasatinib [41]. As described earlier, angiogenesis is an important mechanism in MM, which suggests the potential therapeutic use of BVZ and multitargeted TK's, pazopanib, or sorafenib [34]-[36]. Among the most frequently inactivated tumor suppressor genes in MM is cyclin-dependent kinase inhibitor 2A/alternative reading frame inactivation $(C D K N 2 A / A R F)$. Loss of activity of this gene inactivates tumor suppressing pathways of $p 53$ and retinoblastoma. PB activates $p 53$ and retinoblastoma pathways through inhibition of cyclin-dependent kinases 2 and 4 (CDK2/4) and cyclins E and D3 [38].

MM cells frequently carry mutations of the neurofibromatosis type 2 (NF2) gene [42] [43]. The NF2 product, merlin, down-regulates mTOR component 1 (mTORC1) [44] [45]. This indicates the therapeutic use of mTOR inhibitors sirolimus and everolimus [44]. In addition to genetic changes, epigenetic alterations are involved in the progression of MM [46] [47]. PB and vorinostat can address these abnormalities when added to therapeutic combinations [37] [38].

Results with personalized targeted therapy in patients treated for MM and data emanating from research on molecular mechanisms in this cancer permitted us to propose the following strategy for improving survival of 
patients suffering from advanced MM (Figure 4 and Figure 5). Interruption of two crucial signaling pathways: RAF-MEK-ERK and PI3K-AKT plays a very important part in the successful treatment in MM. This can be accomplished through the combination of erlotinib, pazopanib, BVZ, dasatinib, and PB. Alternatively, pazopanib, lapatinib or trastuzumab can be used in patients with amplification of HER-2. Sorafenib can replace pazopanib and PB may be substituted by ANP. Another important aspect is mTOR and mTOR negative feedback loop signaling. Merlin, a product of oncogene NF2, plays an important part in down-regulation of mTOR, but abnormal integrin $\alpha 5 / \beta 1$ and CD44 and mutation on NF2 negate this action. This can be overcome by everolimus or sirolimus. Increased signaling through the pathways leads to dysregulated cell cycle, cancerous cell metabolism, inhibition of apoptosis, and maintenance and functions of CSC. These complex mechanisms can be controlled by PB, everolimus, and vorinostat or alternatively by ANP, sirolimus, and bortezomib (Figure 5).

The combination therapy with PB, selected targeted agents and/or chemotherapy appears to provide another option for improved survival in patients with advanced MM. The dose reduction of these medications in combination can help avoid serious adverse drug experiences. We are reporting the results of the treatment of a small series of patients who were consecutively admitted for the treatment at BC over the last few years. The strategy was to construct a treatment plan based on molecular profiling when this was obtainable. This resulted in some patients being treated with PB as monotherapy, which in one case contributed to excellent control of the disease (Patient 1). Results obtained in the retrospective evaluation indicate that it was possible to obtain a complete response, stabilization of the disease, and longer median OS compared to other treatment modalities for such patients. We recognize that our findings are preliminary and should be confirmed by well-designed clinical trials.

\section{Conclusion}

Combination chemotherapy with pemetrexed and cisplatin has become the standard-of-care for advanced and

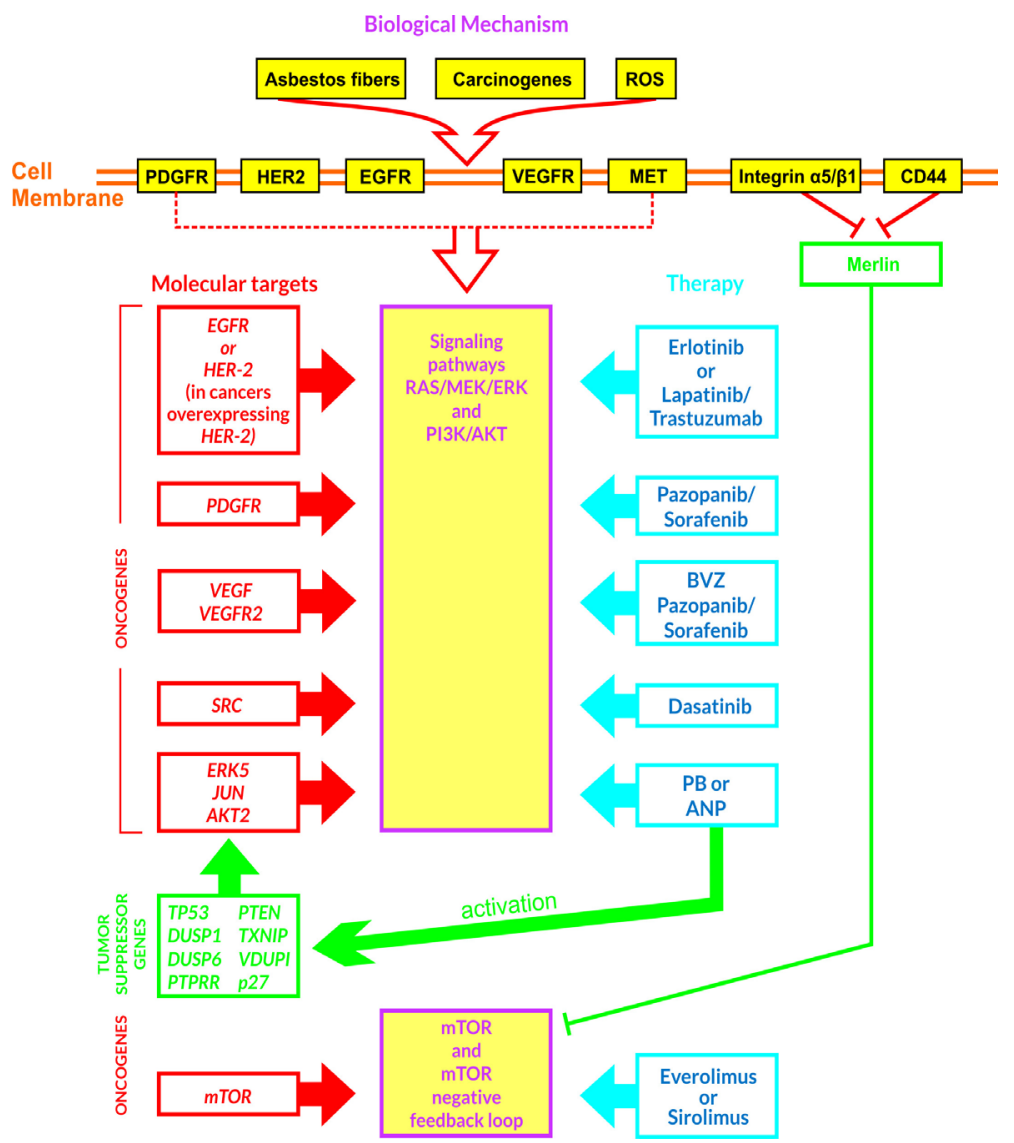

Figure 4. Interruption of signal transduction pathways by PB and targeted agents. 


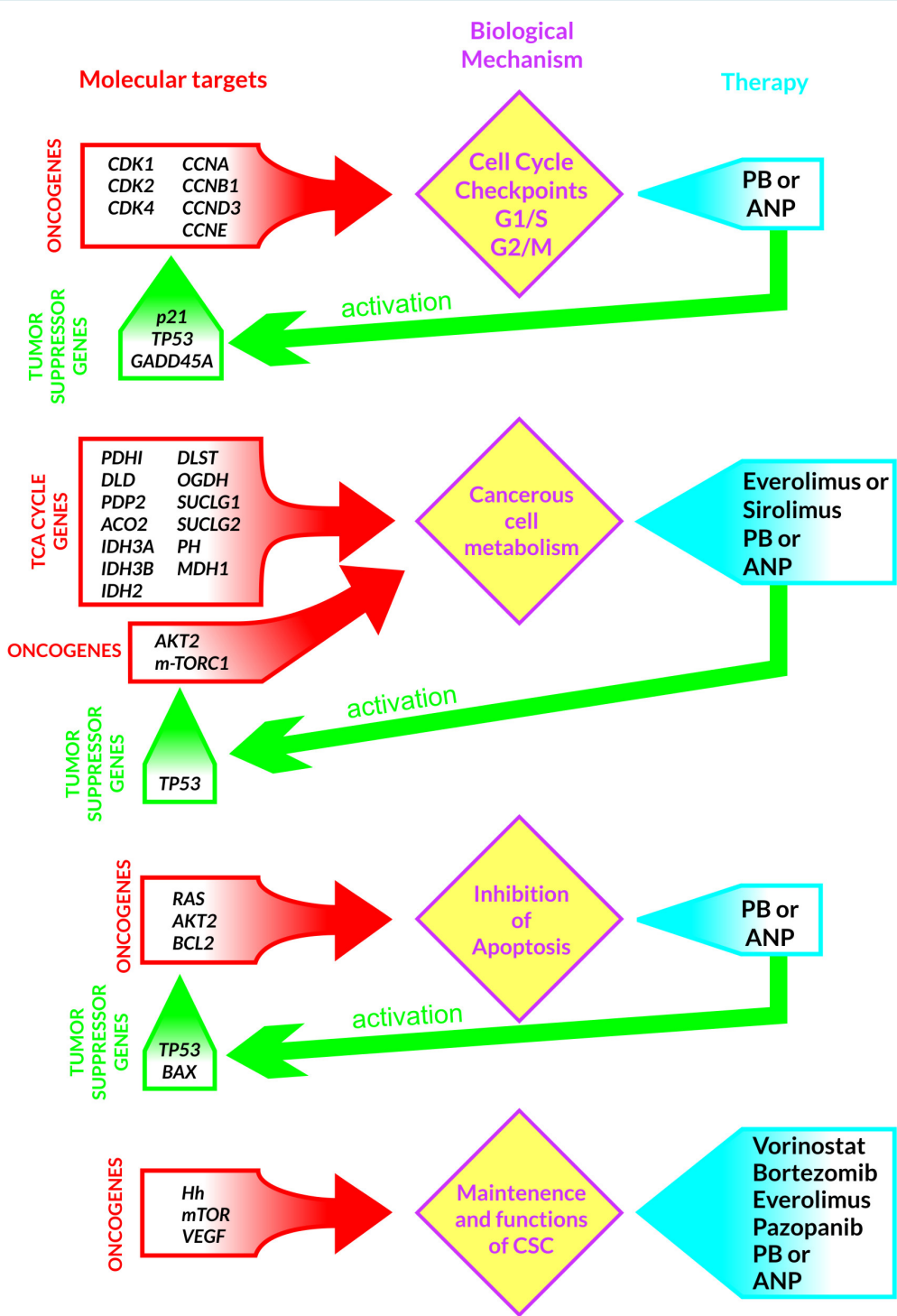

Figure 5. Inhibition of cell cycle, metabolism, maintenance and function of CSS and promotion of apoptosis by PB and targeted agents.

unresectable pleural MM. Targeted therapy carries more promise, but only occasionally stabilizes the disease and is not yet recommended as standard treatment. This retrospective evaluation shows substantial increase of OS and tolerable toxicity compared to other available treatments. Median OS is substantially higher compared to other treatment regimens. The authors realize that these are initial findings and should be validated by a welldesigned phase I/II clinical trial with PB or ANP in combination with targeted agents. We advise caution in the use of these combinations, since clinical trials have not yet been conducted to validate this approach. We an-ticipate that future clinical trials based on molecular profiling will help select a subgroup of cases of advanced MM and correlate the treatment response with genomic changes.

\section{Competing Interests}

All authors are employed by Burzynski Clinic. Dr. Stanislaw R. Burzynski and Dr. Gregory S. Burzynski are shareholders and directors, and Dr. Tomasz J. Janicki is the vice-president of Burzynski Research Institute, Inc. Dr. Stanislaw R. Burzynski is president of Burzynski Research Institute, Inc. Dr. Gregory S. Burzynski is vicepresident of Burzynski Clinic and Dr. Sheldon Brookman is director of Pharmaceutical Development of Burzynski Clinic. 


\section{Acknowledgements}

The authors express their appreciation to the additional physicians involved in the care of the patients: Drs. Zanhua Yi, Alejandro Marquis, Robert Weaver, Sheryl Acelar, Lourdes De Leon, Eva Kubove and Mohammad Khan. Preparation of the manuscript was provided by Carolyn Powers, Jennifer Pineda and Adam Golunski.

\section{References}

[1] Sekido, Y. (2013) Molecular Pathogenesis of Malignant Mesothelioma. Carcinogenesis, 34, 1413-1419. http://dx.doi.org/10.1093/carcin/bgt166

[2] Robinson, B.W.S. and Lake, R.A. (2005) Advances in Malignant Mesothelioma. New England Journal of Medicine, 353, 1591-1603. http://dx.doi.org/10.1056/NEJMra050152

[3] Vogelzang, N.J., Rusthoven, J.J., Symanowski, J., Denham, C., Kaukel, E., et al. (2003) Phase III Study of Pemetrexed in Combination with Cisplatin versus Cisplatin Alone in Patients with Malignant Pleural Mesothelioma. Journal of Clinical Oncology, 21, 2636-2644. http://dx.doi.org/10.1200/JCO.2003.11.136

[4] Sekido, Y. (2011) Inactivation of Merlin in Malignant Mesothelioma Cells and the Hippo Signaling Cascade Dysregulation. Pathology International 61, 331-344. http://dx.doi.org/10.1111/j.1440-1827.2011.02666.x

[5] Zucali, P.A., Ceresoli, G.L., De Vincenzo, F., Simonelli, M., Lorenzi, E., et al. (2011) Advances in the Biology of Malignant Pleural Mesothelioma. Cancer Treatment Reviews, 37, 543-558. http://dx.doi.org/10.1016/j.ctrv.2011.01.001

[6] Jean, D., Daubriac, J., Le Pimpec-Barthes, F., Galateau-Salle, F. and Jaurand, M.-C. (2012) Molecular Changes in Mesothelioma with an Impact on Prognosis and Treatment. Archives of Pathology \& Laboratory Medicine, 136, 277293. http://dx.doi.org/10.5858/arpa.2011-0215-RA

[7] Mossman, B.T., Shukla, A, Heintz, N.H., Verschraegen, C.F., Thomas, A. and Hassan, R. (2013) New Insights into Understanding the Mechanisms, Pathogenesis, and Management of Malignant Mesotheliomas. American Journal of Pathology, 182, 1065-1077. http://dx.doi.org/10.1016/j.ajpath.2012.12.028

[8] Greillier, L., Marco, S. and Barlesi, F. (2011) Targeted Therapies in Malignant Pleural Mesothelioma: A Review of Clinical Studies. Anti-Cancer Drugs, 22, 199-205. http://dx.doi.org/10.1097/CAD.0b013e328341ccdd

[9] Burzynski, S.R. (1969) Investigations on Unknown Ninhydrin-Reacting Substances in Human Blood Serum. I. Attempts at Identification of Three Such Substances. Experientia, 25, 490-491. http://dx.doi.org/10.1007/BF01900774

[10] Burzynski, S.R. (1976) Antineoplastons: Biochemical Defense against Cancer. Physiological Chemistry and Physics, 8, 275-279.

[11] Burzynski, S.R. (1986) Antineoplastons-History of the Research (I). Drugs under Experimental and Clinical Research, 12, 1-9.

[12] Burzynski, S.R. (1986) Synthetic Antineoplastons and Analogs. Drugs of the Future, 11, 679-688.

[13] Burzynski, S.R. (2004) The Present State of Antineoplaston Research (1). Integrative Cancer Therapies, 3, 47-58. http://dx.doi.org/10.1177/1534735403261964

[14] Burzynski, S.R. (2006) Treatments for Astrocytic Tumors in Children: Current and Emerging Strategies. Pediatric Drugs, 8, 167-168. http://dx.doi.org/10.2165/00148581-200608030-00003

[15] Burzynski, S.R., Janicki, T.J., Burzynski, G.S. and Marszalek, A. (2014) The Response and Survival of Children with Recurrent Diffuse Intrinsic Pontine Glioma Based on Phase II Study of Antineoplaston A10 and AS2-1 in Patients with Brainstem Glioma. Childs Nervous System, Published Online. http://dx.doi.org/10.1007/s00381-014-2401-z

[16] Burzynski, S.R., Janicki, T.J., Burzynski, G.S. and Marszalek, A. (2014) A Phase II Study of Antineoplastons A10 and AS2-1 in Children with High-Grade Glioma. Final Report and Review of Recent Trials. Journal of Cancer Therapy, 5, 565-577. http://dx.doi.org/10.4236/jct.2014.56065

[17] Burzynski, S.R., Janicki, T.J. and Burzynski, G.S. (2014) A Phase II Study of Antineoplastons A10 and AS2-1 in Adult Patients with Recurrent Glioblastoma Multiforme. Final Report (Protocol BT-21). Journal of Cancer Therapy, 5, 946-956. http://dx.doi.org/10.4236/jct.2014.510100

[18] Burzynski, S.R., Janicki, T.J., Burzynski, G.S., Marszalek, A. and Brookman, S. (2014) A Phase II Study of Antineoplastons A10 and AS2-1 in Children with Recurrent, Refractory or Progressive Primary Brain Tumors. Final Report (Protocol BT-22). Journal of Cancer Therapy, 5, 977-988. http://dx.doi.org/10.4236/jct.2014.510102

[19] Brusilow, S.W., Danney, M., Waber, L.J., Batshaw, M., Burton, B., et al. (1984) Treatment of Episodic Hyperammonemia in Children with Inborn Errors of Urea Synthesis. New England Journal of Medicine, 310, 1630-1634. http://dx.doi.org/10.1056/NEJM198406213102503

[20] Phuphanich, S., Baker, S.D., Grossman, S.A., Carson, K.A., Gilbert, M.R., et al. (2005) Oral Sodium Phenylbutyrate in Patients with Recurrent Malignant Gliomas: A Dose Escalation and Pharmacologic Study. Neuro-Oncology, 7, 177- 


\section{2. http://dx.doi.org/10.1215/S1152851704000183}

[21] Burzynski, S.R. and Patil, S. (2014) The Effect of Antineoplastons A10 and AS2-1 and Metabolites of Sodium Phenylbutyrate on Gene Expression in Glioblastoma Multiforme. Journal of Cancer Therapy, 5, 929-945. http://dx.doi.org/10.4236/jct.2014.510099

[22] Burzynski, S.R., Burzynski, G.S. and Janicki, T.J. (2014) Recurrent Glioblastoma Multiforme, a Strategy for Long-Term Survival. Journal Cancer Therapy, 5, 957-976. http://dx.doi.org/10.4236/jct.2014.510101

[23] Burzynski, S.R., Janicki, T.J., Burzynski, G.S. and Brookman, S. (2014) Preliminary Findings on the Use of Targeted Therapy in Combination with Sodium Phenylbutyrate in Recurrent Advanced Pancreatic Cancer-A Potential Strategy for Improved Survival. Journal Cancer Therapy, in Press.

[24] Middleton, G.W., Smith, I.E., O’Brien, M.E.R., Norton, A., Hickish, T., Priest, K., et al. (1998) Good Symptom Relief with Palliative MVP (Mitomycin-C, Vinblastine and Cisplatin) Chemotherapy in Malignant Mesothelioma. Annals of Oncology, 9, 269-273. http://dx.doi.org/10.1023/A:1008236010868

[25] Nowak, A.K., Byrne, M.J., Williamson, R., Ryan, G., Segal, A., Fielding, D., et al. (2002) A Multicentre Phase II Study of Cisplatin and Gemcitabine for Malignant Mesothelioma. British Journal of Cancer, 87, 491-496. http://dx.doi.org/10.1023/A:1008236010868

[26] Scherpereel, A., Astoul, P., Baas, P., Berghmans, T., Clayson, H., De Vuyst, P., et al. (2010) Guidelines of the European Respiratory Society and the European Society of Thoracic Surgeons for the Management of Malignant Pleural Mesothelioma. European Respiratory Journal, 35, 479-495. http://dx.doi.org/10.1183/09031936.00063109

[27] Dazzi, H., Hasleton, P.S., Thatcher, N., Wilkes, S., Swindell, R. and Chatterjee, A.K. (1990) Malignant Pleural Mesothelioma and Epidermal Growth Factor Receptor (EGFR). Relationship of EGFR with Histology and Survival Using Fixed Paraffin Embedded Tissue and the F4, Monoclonal Antibody. British Journal of Cancer, 61, 924-926. http://dx.doi.org/10.1038/bjc.1990.207

[28] Cortese, J.F., Gowda, A.L., Wali, A., Eliason, J.F., Pass, H.I. and Everson, R.B. (2006) Common EGFR Mutations Conferring Sensitivity to Gefitinib in Lung Adenocarcinoma Are Not Prevalent in Human Malignant Mesothelioma. International Journal of Cancer, 118, 521-522.

[29] Garland, L.L., Rankin, C., Gandara, D.R., Rivkin, S.E., Scott, K.M., Nagle, R.B., et al. (2007) Phase II Study of Erlotinib in Patients with Malignant Pleural Mesothelioma: A Southwest Oncology Group Study. Journal of Clinical Oncology, 25, 2406-2413. http://dx.doi.org/10.1200/JCO.2006.09.7634

[30] Jackman, D.M., Kindler, H.L., Yeap, B.Y., Fidias, P., Salgia, R., Lucca, J., et al. (2008) Erlotinib plus Bevacizumab in Previously Treated Patients with Malignant Pleural Mesothelioma. Cancer, 113, 808-814. http://dx.doi.org/10.1002/cncr.23617

[31] Mathy, A., Baas, P., Dalesio, O. and Van Zandwijk, N. (2005) Limited Efficacy of Imatinib Mesylate in Malignant Mesothelioma: A Phase II Trial. Lung Cancer, 50, 83-86. http://dx.doi.org/10.1016/j.lungcan.2005.04.010

[32] Porta, C., Mutti, L. and Tassi, G. (2007) Negative Results of an Italian Group for Mesothelioma (g.I.Me.) Pilot Study of Single-Agent Imatinib Mesylate in Malignant Pleural Mesothelioma. Cancer Chemotherapy and Pharmacology, 59, 149-150. http://dx.doi.org/10.1007/s00280-006-0243-4

[33] Dudek, A., Pang, H., Kratzke, R.A., Otterson, G.A., Vokes, E.E. and Kindler, H.L. (2010) CALGB 30601: A Phase II Study of Dasatinib (D) in Patients (pts) with Previously Treated Malignant Mesothelioma (MM) (Abstract 7037). Journal of Clinical Oncology, 28.

[34] Dowell, J.E., Lan, C., Gerber, D., Hughes, R., Yan, J., Xie, Y., et al. (2009) Pemetrexed, Cisplatin and Bevacizumab in Patients with Advanced Malignant Mesothelioma: A Multi-Center Phase II Trial (Abstract b1.2). Journal of Thoracic Oncology, 4, S319.

[35] Nowak, A.K., Millward, M., Francis, R.J., Hasani, A., Van Der Schaaf, A.A., Seguard, T., et al. (2010) Final Results of a Phase II Study of Sunitinib as Second-Line Therapy in Malignant Pleural Mesothelioma (MPM) (Abstract 7036). Journal of Clinical Oncology, 28.

[36] Janne, P.A., Wang, X.F., Krug, L.M., Hodgson, L., Vokes, E.E. and Kindler, H.L. (2007) Sorafenib in Malignant Mesothelioma (MM): A Phase II Trial of the Cancer and Leukemia Group B (CALGB 30307) (Abstract 7707). Journal of Clinical Oncology, 25.

[37] Kelly, W.K., O’Connor, O.A., Krug, L.M., Chiao, J.H., Heaney, M., Curley, T., et al. (2005) Phase I Study of an Oral Histone Deacetylase Inhibitor, Suberoylanilide Hydroxamic Acid, in Patients with Advanced Cancer. Journal of Clinical Oncology, 23, 3923-3931. http://dx.doi.org/10.1200/JCO.2005.14.167

[38] Burzynski, S.R., Burzynski, M.D. and Patil, S. (2014) The Effect of Antineoplastons A10 and AS2-1 and Metabolites of Sodium Phenylbutyrate on Gene Expression in Glioblastoma Multiforme. Journal of Cancer Therapy, 5, 929-945. http://dx.doi.org/10.4236/jct.2014.510099 
[39] Brevet, M., Shimizu, S., Bott, M.J., Shukla, N., Zhou, Q., Olshen, A.B., et al. (2011) Coactivation of Receptor Tyrosine Kinases in Malignant Mesothelioma as a Rationale for Combination Targeted Therapy. Journal of Thoracic Oncology, 6, 864-874. http://dx.doi.org/10.1097/JTO.0b013e318215a07d

[40] Varghese, S., Chen, Z., Bartlett, D.L., Pingpank, J.F., Libutti, S.K., Steinberg, S.M., et al. (2011) Activation of the Phosphoinositide-3-Kinase and Mammalian Target of Rapamycin Signaling Pathways Are Associated with Shortened Survival in Patients with Malignant Peritoneal Mesothelioma. Cancer, 117, 361-371. http://dx.doi.org/10.1002/cncr.25555

[41] Menges, C.W., Chen, Y., Mossman, B.T., Chernoff, J., Yeung, A.T. and Testa, J.R. (2010) A Phosphotyrosine Proteomic Screen Identifies Multiple Tyrosine Kinase Signaling Pathways Aberrantly Activated in Malignant Mesothelioma. Genes and Cancer, 1, 493-505. http://dx.doi.org/10.1177/1947601910375273

[42] Thurneysen, C., Opitz, I., Kurtz, S., Weder, W., Stahel, R.A. and Felley-Bosco, E. (2009) Functional Inactivation of NF2/Merlin in Human Mesothelioma. Lung Cancer, 64, 140-147. http://dx.doi.org/10.1016/j.lungcan.2008.08.014

[43] Stamenkovic, I. and Yu, Q. (2010) Merlin, a "Magic” Linker between the Extracellular Cues and Intracellular Signaling Pathways That Regulate Cell Motility, Proliferation and Survival. Current Protein and Peptide Science, 11, 471484. http://dx.doi.org/10.2174/138920310791824011

[44] Lopez-Lago, M.A., Okada, T., Murillo, M.M., Socci, N. and Giancotti, F.G. (2009) Loss of the Tumor Suppressor Gene NF2, Encoding Merlin, Constitutively Activates Integrin-Dependent mTORC1 Signaling. Molecular and Cellular Biology, 29, 4235-4249. http://dx.doi.org/10.1128/MCB.01578-08

[45] James, M.F., Han, S., Polizzano, C., Plotkin, S.R., Manning, B.D., Stemmer-Rachamimov, A.O., et al. (2009) NF2/Merlin Is a Novel Negative Regulator of mTOR Complex 1 and Activation of mTORC1 Is Associated with Meningioma and Schwannoma Growth. Molecular and Cellular Biology, 29, 4250-4261. http://dx.doi.org/10.1128/MCB.01581-08

[46] Christensen, B.C., Houseman, E.A., Godleski, J.J., Marsit, C.J., Longacker, J.L., Roelofs, C.R., et al. (2009) Epigenetic Profiles Distinguish Pleural Mesothelioma from Normal Pleura and Predict Lung Asbestos Burden and Clinical Outcome. Cancer Research, 69, 227-234. http://dx.doi.org/10.1158/0008-5472.CAN-08-2586

[47] Goto, Y., Shinjo, K., Kondo, Y., Shen, L., Toyota, M., Suzuki, H., et al. (2009) Epigenetic Profiles Distinguish Malignant Pleural Mesothelioma from Lung Adenocarcinoma. Cancer Research, 69, 9073-9082.

http://dx.doi.org/10.1158/0008-5472.CAN-09-1595 


\section{Abbreviations}

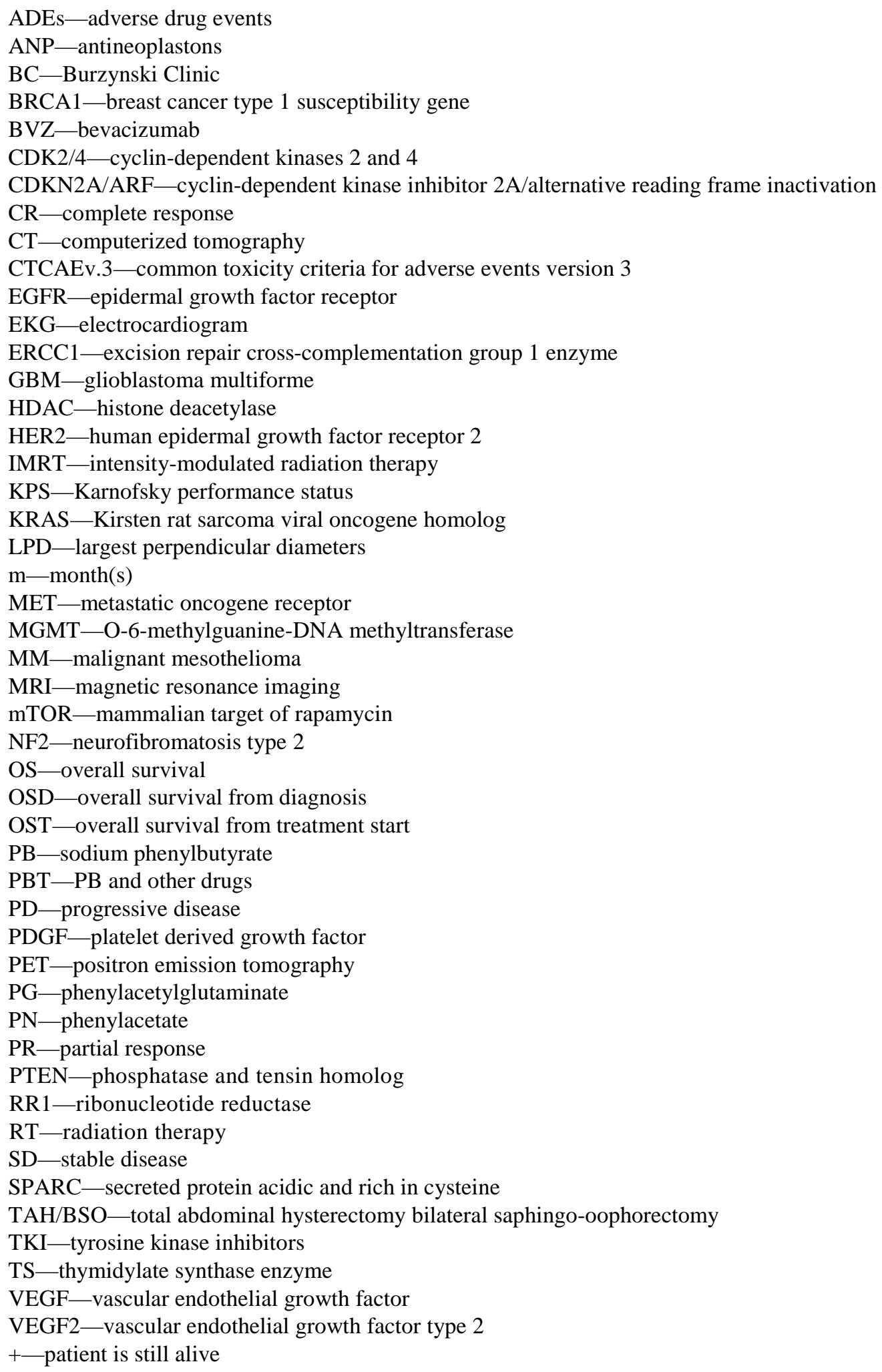


Scientific Research Publishing (SCIRP) is one of the largest Open Access journal publishers. It is currently publishing more than 200 open access, online, peer-reviewed journals covering a wide range of academic disciplines. SCIRP serves the worldwide academic communities and contributes to the progress and application of science with its publication.

Other selected journals from SCIRP are listed as below. Submit your manuscript to us via either submit@scirp.org or Online Submission Portal.
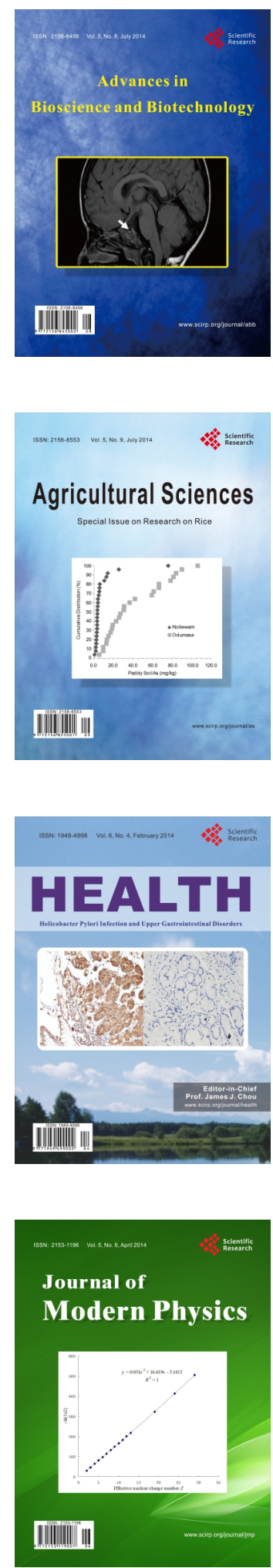
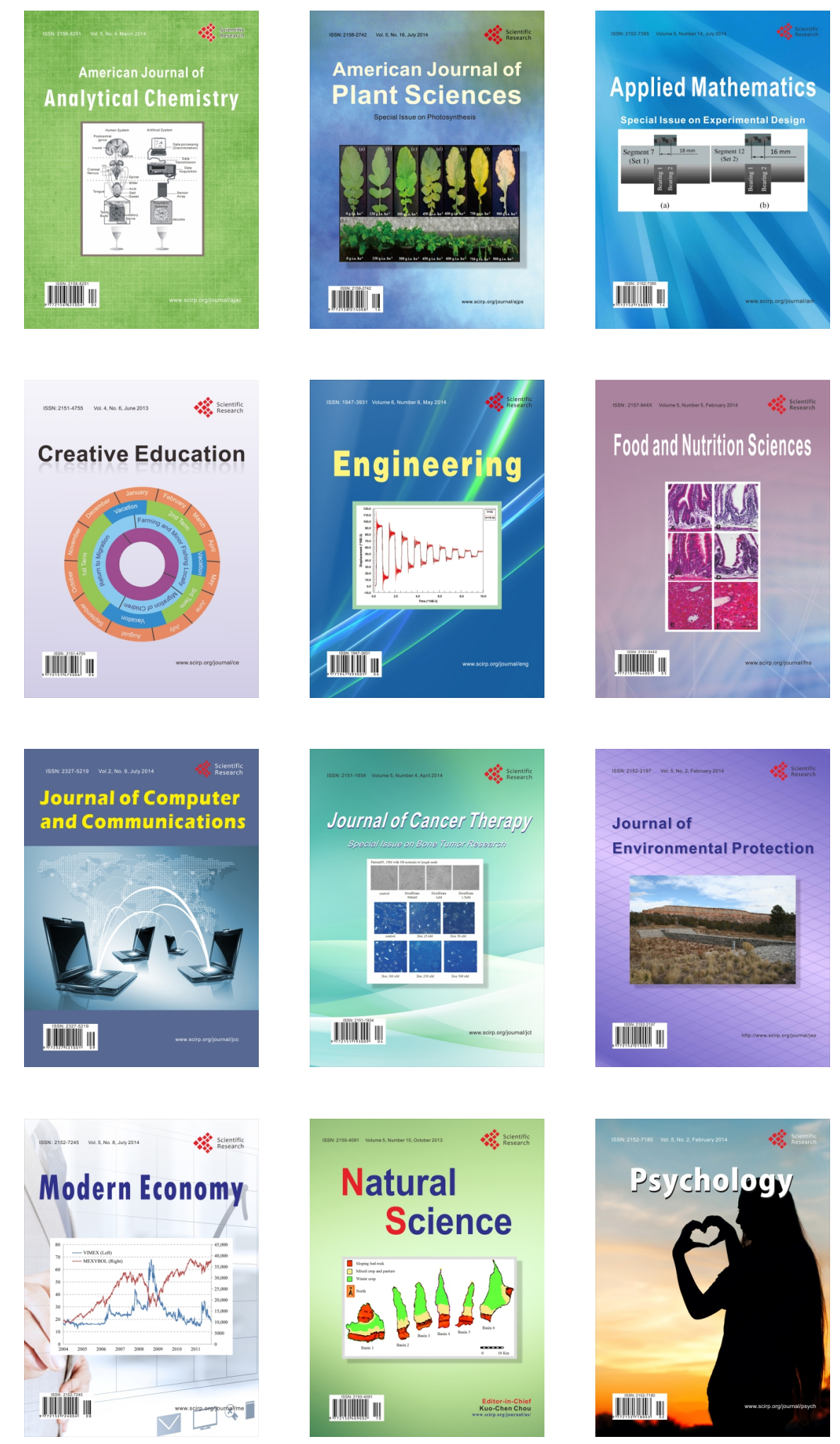